\title{
Microbiota cérvico-vaginal de ovelhas mestiças e sua susceptibilidade aos antibióticos ${ }^{1}$
}

\author{
Valdenice F. Silva ${ }^{2}$, Tácito E.F. Damasceno ${ }^{3}$, Natson J.D. Souza ${ }^{4}$, Isabelle Franco ${ }^{5}$ \\ e Mateus M. Costa $6^{*}$
}

\begin{abstract}
Silva V.F., Damasceno T.E.F., Souza N.J.D., Franco I. \& Costa M.M. 2011. [Cervical-vaginal microbiota of crossbred sheep in Petrolina/PE, Brazil, and its susceptibility to antibiotics.] Microbiota cérvico-vaginal de ovelhas mestiças e sua susceptibilidade aos antibióticos. Pesquisa Veterinária Brasileira 31(7):586-590. Campus Ciências Agrárias, Universidade Federal do Vale do São Francisco, BR 407 Km 12, Lote 543, Projeto de Irrigação Senador Nilo Coelho s/n, Petrolina, PE 56300-990, Brazil. E-mail: mateus.costa@univasf.edu.br

Sheep farming has developed in recent decades; however there is still little information on the composition and pathogenic potential of cervical-vaginal flora of sheep. The purpose of the present study was to determine the main constituents of microorganisms in the cervicalvaginal flora of sheep and their antimicrobial susceptibility. Samples were taken from 60 healthy sheep belonging to herds in the region of Petrolina, Pernambuco. Bacterial isolation was performed on blood agar and MacConkey agar, and the microorganisms were identified according to morphology, Gram staining and biochemical characteristics. The samples were subjected to disk diffusion test for determination of sensitivity to the following antimicrobial drugs: sulfamethazine, enrofloxacin, doxycycline, tetracycline, penicillin, amoxicillin, cephalothin and lincomycin. We obtained 94 isolates and found a higher frequency of Staphylococcus spp., Escherichia coli and Micrococcus spp., and also observed isolates of Acinetobacter spp., Shigella spp., Enterobacter spp., Klebsiella spp. and Streptococcus spp. The isolates were highly sensitive to the antibiotics tested, with the lowest percentage of susceptibility to lincomycin. The presence of opportunistic microorganisms of a potential pathogenic microbiota, such as Staphylococcus spp. and Escherichia coli, refers to a careful analysis in the diagnosis of genital infections. The bacterial isolates obtained in this study are sensitive to most groups of antibiotics tested, demonstrating the potential use of these active ingredients, plus the availability of choice, given the lack of multidrug resistance.
\end{abstract}

INDEX TERMS: Cervical-vaginal microbiota, antimicrobial sensitivity, sheep.

\footnotetext{
${ }^{1}$ Recebido em 23 de novembro de 2010.

Aceito para publicação em 23 de março de 2011.

${ }^{2}$ Mestranda do Curso de Pós-Graduação em Ciência Animal, Campus Ciências Agrárias, Universidade Federal do Vale do São Francisco (UFVSF), Rod. BR 407 Km 12, Lote 543, Projeto de Irrigação Senador Nilo Coelho s/ n, Petrolina, PE 56300-990, Brasil. E-mail: valdenice.felix@hotmail.com

${ }^{3}$ Graduando Medicina Veterinária, Campus Ciências Agrárias, UFVSF, Projeto de Irrigação Senador Nilo Coelho s/n, Petrolina, PE. E-mail: te_damasceno@hotmail.com

${ }^{4}$ Bolsista Iniciação Científica Júnior, Campus Ciências Agrárias, UFVSF, Projeto de Irrigação Senador Nilo Coelho s/n, Petrolina, PE. E-mail: natsonagropecuaria@hotmail.com

${ }^{5}$ Bolsista Fixação Técnica, Campus Ciências Agrárias, UFVSF, Projeto de Irrigação Senador Nilo Coelho s/n. Petrolina, PE. E-mail: isafelinos@hotmail.com

${ }^{6}$ Colegiado Zootecnia, Campus Ciências Agrárias, UFVSF, Projeto de Irrigação Senador Nilo Coelho s/n, Petrolina, PE. *Autor para correspondência: mateus.costa@univasf.edu.br
}

RESUMO.- A criação de ovinos tem se desenvolvido nas últimas décadas, entretanto ainda são escassas informações sobre a composição e potencial patogênico da microbiota cérvico-vaginal de ovelhas. 0 presente estudo teve como objetivo conhecer os microrganismos constituintes da microbiota cérvico-vaginal de ovelhas, bem como sua susceptibilidade aos antimicrobianos. Foram realizadas coletas em 60 animais sadios, pertencentes a rebanhos de Petrolina e região. Foi realizado o isolamento bacteriano em ágar sangue e ágar MacConkey, sendo os microrganismos identificados de acordo com características morfológicas, tintoriais e bioquímicas. As amostras foram submetidas ao teste de difusão em disco para determinar o perfil de sensibilidade aos antimicrobianos: sulfametazina, enrofloxacina, doxiciclina, tetraciclina, penicilina, amoxicilina, cefalotina e lincomicina. Foram obtidos 94 isolados, sendo constatada uma maior fre- 
quência de Staphylococcus spp. (32,97\%), Escherichia coli e Micrococcus spp., sendo observado ainda, isolados de Acinetobacter spp., Shigella spp., Enterobacter spp., Klebsiella spp. e Streptococcus spp. Os isolados apresentaram alta sensibilidade aos antimicrobianos testados sendo observado o menor percentual de sensibilidade para lincomicina. A presença de microrganismos oportunistas de potencial patogênico, na microbiota, como Staphylococcus spp e Escherichia coli, remete a uma análise criteriosa em relação ao diagnóstico de infecções genitais. Os isolados bacterianos obtidos neste estudo são sensíveis à maioria dos grupos de drogas antimicrobianas testadas, demonstrando o potencial de utilização desses princípios ativos, além da disponibilidade de escolha, visto a ausência de multirresistência.

TERMOS DE INDEXAÇÃO: Microbiota cérvico-vaginal, sensibilidade antimicrobiana, ovinos.

\section{INTRODUÇÃo}

A ovinocultura vem crescendo nos últimos anos no Brasil, sendo em grande parte explorada na região nordeste do Brasil. 0 estado de Pernambuco possui cerca de 1,3 milhões de ovinos (IBGE 2009), concentrados principalmente na região do município de Petrolina. As principais causas da baixa eficiência reprodutiva em ovelhas são as infecções do trato reprodutivo causadas por bactérias, principalmente durante o período pósparto, tendo como consequência perdas econômicas com o descarte de matrizes e redução do número de crias (Bouters \& Vandesplassche 1977, Gregory \& Rizzo 2009).

Sob condições normais, a microbiota vaginal apresenta composição e número variável de microrganismos, sendo estes também encontrados na pele e fezes, e devido suas propriedades invasivas, podem estar em pequeno número no útero de animais sadios (Ramaswamy et al. 1991). Os microrganismos habituais da vagina tornam-se patogênicos quando os animais apresentam o sistema imunológico comprometido, em decorrência do estresse causado por fatores variados tais como súbitas mudanças de temperatura, nutrição deficiente, final de gestação e parto, demonstrando seu caráter oportunista na origem de infecções (Verma et al. 1994, Kuntze \& Aurich 1995, Lianjuan et al. 1995). Apesar das fêmeas possuírem barreiras físicas que impedem a colonização do trato genital por patógenos oportunistas, as mesmas podem se tornar susceptíveis aos microrganismos que passam pela cérvix e alcançam o útero (Rocha et al. 2004). Elliot et al. (1968) citam que durante o período pós-parto ocorre a migração dos microrganismos presentes na vulva e vagina para a cérvix e útero. Parte das bactérias isoladas de conteúdo vaginal constitui-se de bastonetes gram negativos provenientes do trato gastrintestinal (TGI), especialmente Escherichia coli e cocos Gram-positivos, como Staphylococcus spp. e Streptococcus spp. (Ramaswamy et al. 1991, Sharda et al. 1991, Balassu et al. 1992, Campero et al. 1992, Arthur et al. 1996, Kunz et al. 2002).

A comunidade científica tem manifestado, nos últimos anos, preocupação em relação à resistência às drogas antimicrobianas, devido suas graves consequências, no tratamento de doenças. Várias publicações científicas relatam a relação entre os antibióticos usados em animais e a seleção de cepas bacterianas resistentes e de importância na gênese de enfermidades nos seres humanos e nos animais (Piddock 1996, Witte 1998, Torres \& Zarazaga 2002, Carneiro et al. 2007, Macedo et al. 2007).

Devido à presença de agentes com potencial oportunista no trato genital e ainda a carência de estudos científicos sobre a utilização de antimicrobianos relacionados às infecções genitais de ovelhas, é de fundamental importância o conhecimento da microbiota aeróbia parcial, bem como a determinação de sua susceptibilidade às drogas antimicrobianas, a fim de que a terapia correta e planejada seja estabelecida.

\section{MATERIAL E MÉTODOS}

Para realização do experimento foram visitadas propriedades com rebanhos ovinos da região de Petrolina, Pernambuco. Os 60 animais utilizados se destinavam à produção de carne, sendo a amostragem constituída por fêmeas mestiças, em idade reprodutiva, de diferente faixa etária, sadias, não-prenhes e criadas em sistema extensivo e semi-extensivo. A sanidade reprodutiva dos animais foi verificada por meio de histórico e exame clínico do aparelho genital.

Amostras da mucosa cérvico-vaginal foram obtidas a partir de swabs esterilizados que após higiene da região vulvar foram introduzidos por meio de espéculo asséptico até o fundo de saco vaginal, realizando-se movimentos de rotação visando obter maior quantidade de material. Após a coleta, os swabs foram acondicionados em tubos esterilizados contendo meio de transporte Stuart modificado e encaminhados ao Laboratório de Microbiologia e Imunologia Animal do Campus de Ciências Agrárias da Universidade Federal do Vale do São Francisco para processamento.

Os swabs foram semeados em ágar-sangue contendo $5 \%$ de sangue desfibrinado de carneiro e ágar MacConkey e incubados em estufa a $37^{\circ} \mathrm{C}$ por 24 horas. Não se empregou metodologia para o isolamento de bactérias anaeróbias. As bactérias foram identificadas de acordo características morfológicas, tintoriais e bioquímicas conforme descrito por Quinn et al. (1994). Para identificação das espécies de Staphylococcus foram utilizados os testes bioquímicos: coagulase, ágar púrpura de bromocresol (PAB), manitol semi-sólido, glicose semi-sólido, DNAse, esculina e urease.

A metodologia utilizada para verificar a sensibilidade dos agentes isolados aos antimicrobianos foi o método de difusão em disco Kirby-Bauer modificado (CLSI 2006). Os cultivos foram repassados para tubos de ensaio contendo caldo Müller-Hinton e incubados a $37^{\circ} \mathrm{C}$ até apresentar uma turbidez equivalente a 0,5 da escala padrão de Mac Farland. Com auxílio de um $s w a b$, os isolados foram semeados em placas de Petri contendo ágar Müller Hinton. A etapa seguinte consistiu na aplicação dos discos, em que uma leve pressão permitiu o contato entre os mesmos e a superfície do meio inoculado. Os agentes antimicrobianos avaliados foram: penicilina $(10 \mu \mathrm{g})$, tetraciclina $(30 \mu \mathrm{g})$, sulfametazina $(300 \mu \mathrm{g})$, doxiciclina $(30 \mu \mathrm{g})$, amoxicilina $(10 \mu \mathrm{g})$, enrofloxacina $(5 \mu \mathrm{g})$, cefalotina $(30 \mu \mathrm{g})$ e lincomicina $(2 \mu \mathrm{g})$. As placas foram incubadas em estufa durante $24 \mathrm{~h}$ a $37^{\circ} \mathrm{C}$. Após a leitura dos halos foi determinado o perfil de sensibilidade dos isolados. Foi determinado o índice de resistência aos antimicrobianos dividindo o número de grupos de antimicrobianos aos quais os isolados foram resistentes pelo total de grupos de antimicrobianos testados (Krumperman 1983), sendo considerada multiresistência valores superiores a 0,4.

\section{RESULTADOS}

Das 60 amostras analisadas foram obtidos 94 isolados bacterianos. Foram observadas as seguintes frequências de microrganismos: Staphylococcus spp. (32,97\%), Escherichia coli (14,90\%), Micrococcus spp. (12,8\%), Acinetobacter spp.

Pesq. Vet. Bras. 31(7):586-590, julho 2011 
Quadro 1. Percentual de sensibilidade aos antimicrobianos de microrganismos isolados da mucosa cérvico vaginal de ovelhas

\begin{tabular}{|c|c|c|c|c|c|c|c|c|c|c|}
\hline Isolados & $(\mathrm{N})$ & SUT & ENO & $\mathrm{CEF}$ & PEN & AMO & DOX & TET & LIN & IRMA \\
\hline S. epidermidis & 06 & 100 & 100 & 100 & 83,3 & 100 & 100 & 66,6 & 83,3 & 0,06 \\
\hline S.saprophyticus & 01 & 100 & 100 & 0 & 0 & 0 & 100 & 100 & 0 & 0,5 \\
\hline S. schleiferi & 19 & 68,4 & 89,4 & 84,2 & 73,6 & 84,2 & 94,7 & 89,4 & 68,4 & 0,14 \\
\hline S.lentus & 1 & 100 & 0 & 100 & 100 & 100 & 100 & 100 & 0 & 0,16 \\
\hline S.arletae & 1 & 100 & 100 & 0 & 0 & 0 & 100 & 0 & 0 & 0,5 \\
\hline Staphylococcus spp. & 2 & 100 & 100 & 100 & 50 & 50 & 100 & 100 & 50 & 0,33 \\
\hline Escherichia coli & 14 & 92,8 & 92,9 & 0 & 0 & 78,6 & 92,9 & 78,6 & 0 & 0,36 \\
\hline Micrococcus spp. & 12 & 41,7 & 91,7 & 66,7 & 41,7 & 50 & 100 & 75 & 41,7 & 0,30 \\
\hline Acinetobacter spp. & 11 & 70 & 70 & 70 & 70 & 90 & 100 & 70 & 30 & 0,25 \\
\hline Enterobacter spp. & 10 & 80 & 80 & 20 & 0 & 100 & 90 & 100 & 0 & 0,33 \\
\hline Klebsiella spp. & 10 & 80 & 90 & 90 & 20 & 90 & 100 & 90 & 20 & 0,26 \\
\hline Streptococcus spp. & 7 & 71,4 & 57,1 & 42,9 & 42,9 & 71,4 & 100 & 100 & 0 & 0,26 \\
\hline Shigella spp. & 1 & 100 & 100 & 0 & 0 & 100 & 100 & 0 & 0 & 0,50 \\
\hline Média sensibilidade (\%) & & 84,9 & 82,3 & 51,8 & 37,0 & 70,3 & 98,2 & 74,6 & 22,5 & \\
\hline
\end{tabular}

$\mathrm{N}$ = número de isolados, $\mathrm{SUT}=$ sulfametazina, $\mathrm{ENO}=$ enrofloxacina, $\mathrm{CEF}=$ cefalotina, $\mathrm{PEN}=$ penicilina, $\mathrm{AMO}=$ amoxicilina, $\mathrm{DOX}=$ doxiciclina, TET $=$ tetraciclina, LIN = lincomicina.

$(10,6 \%)$, Enterobacter spp. $(10,64 \%)$, Klebsiella spp. $(9,6 \%)$, Streptococcus spp. $(7,5 \%)$ e Shigella spp. (1,1\%). No gênero Staphylococcus foram identificadas as seguintes espécies: $S$. schleiferi (63,3\%), S. epidermidis (20,0\%), S. saprophyticus, $S$. arlettae e $S$. lentus respectivamente com 3,3\% e Staphylococcus spp. $(6,7 \%)$, em que não foi possível a identificação com as provas bioquímicas realizadas.

0 perfil de sensibilidade dos isolados aos antimicrobianos está demonstrado no Quadro 1.0 gênero Staphylococcus apresentou melhor sensibilidade à doxiciclina e enrofloxacina com percentuais de 93,8\% e 90,6\%, respectivamente. Micrococcus spp. demonstraram sensibilidade de $100 \%$ à doxiciclina obtendo menores percentuais à sulfametazina, penicilina e lincomicina. Streptococcus spp. mostraram-se 100\% sensível à doxicilina e tetraciclina, e menores percentuais referentes à cefalotina, penicilina e lincomicina com valores de $42,9 \%$ e $0,0 \%$, respectivamente.

Escherichia coli apresentou maior sensibilidade à sulfametazina, enrofloxacina e doxiciclina $(92,9 \%)$ e resistência à cefalotina, penicilina e lincomicina. Resultados semelhantes foram encontrados para Enterobacter spp., que foram menos sensível às mesmas drogas, com melhor percentual para amoxicilina, tetraciclina (100\%) e doxiciclina (90\%). Acinetobacter spp. obtiveram percentual de $100 \%$ à doxiclina, sendo a sensibilidade mais baixa relacionada à lincomicina (30\%). Klebsiella spp. apresentaram alta sensibilidade à maioria dos antimicrobianos, obtendo menor percentual à penicilina e lincomicina (20\%). Shigella spp. apresentaram sensibilidade de $100 \%$ às drogas sulfametazina, enrofloxacina, amoxicilina e doxiciclina. Em média a maioria dos antimicrobianos testados apresentou um alto percentual de sensibilidade, tendo a penicilina e lincomicina alcançado os menores valores. 0 índice de resistência múltipla dos isolados variou de 0,15 a 0,5 entre os gêneros testados, sendo os índices obtidos respectivamente para isolados de Staphylococcus spp. e Shigella spp.

\section{DISCUSSÃO}

Os principais microrganismos isolados neste estudo foram Staphylococcus spp. e Escherichia coli, o que corrobora com estudos semelhantes sobre o levantamento da microbiota presente na mucosa cérvico-vaginal de fêmeas domésticas (Rocha et al. 2004, Andrade 2006, Gomes 2006). Concorda ainda com outros autores que afirmam que em condições normais a composição e o número de agentes da microbiota são variáveis (Ramaswamy et al. 1991, Sharda et al.1991, Balassu et al. 1992, Campero et al. 1992, Arthur et al. 1996, Kunz et al. 2002).

Microrganismos como Streptococcus spp., Staphylococcus spp. e coliformes são considerados habitantes da região vulvar, vagina, pele e trato gastrintestinal e possuem propriedades invasivas nos tecidos (Oliveira 1995). Streptococcus spp., quando associado a outros microrganismos, pode favorecer a proliferação de bactérias de maior patogenicidade, pois estes produzem penicilinase e protegem outras bactérias (Panangala et al. 1978, Kreplin 1990).

A predominância de Staphylococcus spp. deve-se possivelmente a este microrganismo ser originário da pele e mucosas, uma vez que são encontrados comumente em outros ruminantes (Rocha et al. 2004). Em um estudo da microbiota vaginal de vacas associadas a infecções uterinas, Gani et al. (2008) verificaram a predominância do gênero Staphylococcus spp., corroborando com os dados descritos na literatura. Relatos semelhantes foram descritos em ovelhas por Moorthy \& Singh (1982). Martins et al. (2009) verificaram a prevalência de Staphylococcus spp. coagulase positiva na mucosa cérvico-vaginal de ovelhas. Entretanto, S. epidermidis foi isolado de seis animais, corroborando com os achados do presente estudo. $\mathrm{O}$ isolamento de S. schleiferi na mucosa genital já foi descrita em algumas espécies como em jabutis (Pessoa 2009), cães (Coggan et al. 2008) e em humanos (Campos et al. 2008), entretanto são escassos estudos referentes a este microrganismo no sistema genital de ovelhas. Bactérias do gênero Micrococcus são descritas como habitantes das mucosas de diversas espécies animais (Gomes 2006, Fornazari et al. 2007, Uchôa et al. 2009), sendo considerado de baixa patogenicidade (Wunder et al. 1976). 0 isolamento deste microrganismo nos animais do presente estudo foi esperado, contudo dada a ausência de patogenicidade do mesmo, este deve ser diferenciado de outros cocos Gram-positivos de maior potencial patogênico.

Segundo Kuntze \& Aurich (1995), as enterobactérias predominantes na mucosa da vulva e vagina são oriundas do trato gastrintestinal e podem causar processos infecciosos e deslocamento de células inflamatórias para os tecidos. No presente estudo, os principais gêneros de enterobactérias foram isola- 
dos, demonstrando a importância desses microrganismos na composição da microbiota genital de ovelhas e o risco de desenvolvimento de enfermidades reprodutivas, principalmente no pós- parto, quando a cérvix está relaxada, facilitando a invasão desses agentes oportunistas. Husted et al. (2003) em um levantamento de microbiota vaginal comparando vacas sadias e com vaginite, descreveram um percentual de $33 \%$ para $E$. coli e 20\% para Staphylococcus spp. Entretanto Acinetobacter wolffii foi o microrganismo predominante em ambos os grupos, apresentando um percentual de $95 \%$, enquanto que no presente estudo o percentual desse gênero foi de 10,64\%. A alta prevalência do microrganismo tanto em animais sadios como em enfermos o caracteriza como primário ou secundário da infecção, todavia são escassas informações sobre esse agente como constituinte da microbiota de ovelhas, sendo isolado nessa espécie principalmente em casos de mastite (Domingues et al. 2006, Drescher et al. 2010).

É de suma importância o conhecimento da microbiota dos animais sadios para que esta possa ser comparada com resultados de culturas obtidas de animais doentes. 0 presente estudo verificou a presença de microrganismos considerados de baixa patogenicidade, mas também agentes de caráter oportunista, que em situações de desequilíbrio podem desencadear doenças reprodutivas. 0 oportunismo dessas infecções é decorrente do manejo que é aplicado às fêmeas, principalmente o manejo alimentar no pós-parto, em que o animal se apresenta mais susceptível às doenças. Torres et al (1997) observaram associação entre estresse e a ocorrência de infecções bacterianas no útero de vacas leiteiras, principalmente durante o período pós-parto. A microbiota normal residente protege o hospedeiro contra a colonização por essas bactérias potencialmente patogênicas. Esse antagonismo ocorre por meio da competição por nutrientes, produção de substâncias nocivas, alteração de $\mathrm{pH}$ e disponibilidade de oxigênio, reduzindo dessa forma a proliferação dos patógenos (Tabulsi 1999).

Em relação à sensibilidade aos antimicrobianos, observouse alta sensibilidade dos isolados às drogas antimicrobianas utilizadas neste estudo. 0 menor percentual de sensibilidade foi encontrado para a lincomicina e penicilina. Segundo Aiello (2001), a lincomicina possui um espectro limitado contra patógenos aeróbios, sendo os microrganismos gram negativos resistentes. 0 autor ainda ressalta que a resistência à lincomicina surge lentamente talvez como resultado de uma mutação cromossômica, podendo exibir resistência cruzada com outros antibióticos in vitro. Streptococcus spp. isolados de vacas com mastite foram resistentes à lincomicina (Faublée et al. 2002). Segundo os autores, o fenótipo comum envolvido na resistência em cocos gram positivos é o $\mathrm{MLS}_{\mathrm{B}}$, com resistência cruzada entre macrolídeos, lincosamidas e estreptograminas do grupo $\mathrm{B}$, que ocorre devido à metilação do $23 \mathrm{~S}$ do rRNA, resultando em diminuição da afinidade para drogas antimicrobianas destes grupos.

A baixa sensibilidade dos microrganismos frente à penicilina já foi demonstrada em artigos científicos (Riedner et al. 1987, Fthenakis 1998, Freitas et al. 2005, Machado et al. 2008). A resistência aos â-lactâmicos, principalmente pelos gram positivos, pode ser oriunda tanto pela alteração das proteínas de ligação à droga quanto pela produção de â-lactamases (Lyon et al. 1987, Livermore et al. 1995). Jacob et al (2002) avaliando a susceptibilidade de bactérias isoladas de swab uterino e da fossa clitoriana de éguas, observaram um percentual médio de sensibilidade à penicilina de $29,16 \%$ e $16,6 \%$ respectivamente. Neste mesmo estudo, as bactérias Gram negativas foram totalmente resistentes a esta droga, sendo esses achados semelhantes aos descritos aqui. Apesar da pressão de seleção oriunda da utilização massiva da droga constantemente relatada na literatura, os gêneros gram positivos apresentaram um percentual de sensibilidade relativamente boa frente à penicilina, podendo este fármaco ser utilizado no tratamento de infecções causadas por esses agentes. Bactérias gram negativas apresentam resistência intrínseca à penicilina, principalmente as enterobactérias, que no presente estudo foram os microrganismos isolados em maior número, justificando dessa forma a baixa efetividade dessa droga.

Martins et al. (2009) avaliando a susceptibilidade de bactérias isoladas da mucosa vaginal de ovelhas observaram que os coliformes apresentaram baixa sensibilidade à amoxicilina, tetraciclina e cefalotina, não corroborando com os achados deste estudo, que verificou alta sensibilidade os isolados frente às mesmas drogas. 0 perfil de sensibilidade dos microrganismos frente aos antimicrobianos pode variar de acordo com a região em que o estudo foi realizado, visto que o presente experimento foi realizado no nordeste e aquele no sudeste do país onde as condições de produção de ovinos e a terapia de infecções podem ser distintas.

Perante o número de drogas antimicrobianas testadas neste estudo, e cálculo de IRMA segundo Krumperman (1983), os gêneros de microrganismos não se apresentaram multirresistentes. Porém, um isolado de Enterobacter spp. apresentou um índice de 0,83. A multirresistência dos microrganismos é oriunda do uso inadequado das drogas antimicrobianas, apresentando um risco potencial para a saúde pública que pode dificultar o tratamento de doenças animais e de humanos, agravando quadros clínicos curáveis (Sena 2000).

\section{CONCLUSÕES}

O conhecimento acerca da microbiota cérvico-vaginal de ovelhas é de suma importância, visto que infecções genitais são causas de baixa produção na exploração dessa espécie.

A presença de microrganismos oportunistas de potencial patogênico, como Staphylococcus spp. e Escherichia coli, remete a uma análise criteriosa em relação ao diagnóstico e terapêutica das infecções do trato reprodutivo.

Os isolados bacterianos obtidos neste estudo são sensíveis à maioria dos grupos de drogas antimicrobianas testadas, demonstrando o potencial de utilização desses princípios ativos, além da disponibilidade de escolha, visto a ausência de multirresistência.

\section{REFERÊNCIAS}

Aiello S.E. 2001. Manual Merck de Veterinária. 8ª ed. Roca, São Paulo. 1861p. Andrade J.B. 2006. Estudo microbiológico e citológico do trato genital de gatas domésticas. Dissertação de Mestrado, Universidade Federal Rural do Rio de Janeiro, Seropédica, RJ. 43p.

Arthur G.H., Noakes D.E., Pearson H. \& Parkinson T.J. 1996. Veterinary Reproduction and Obstetrics. $1^{\text {th }}$ ed. W.B. Saunders Company, London.

Balassu M.T., Torres E.B. \& Vizmanos M.F.C. 1992. Bacteriologic profile of the uterus and vagina of non-pregnant buffalo-cows. Journal of Medicine, Philadelphia, 29(2):35-41. 
Bouters R. \& Vandeplassche M. 1977. Post partum infection in cattle: Diagnosis, preventive and curative treatment. J. South Afr. Vet. Assoc. 48(4):237-239.

Campero C.M., Conosciuto G. \& Odriozola E. 1992. Hallazgos clinicos, bacteriológicos e histopathologicos en vaca lecheras, asociados com problemas reprodutivos. Revta Med. Vet., Buenos Aires, 72(6):264-272.

Campos A.C.C., Junior R.F., Ribeiro L.F.J., Paulinelli R.R. \& Reis C. 2008. Prevalence of vulvovaginitis and bacterial vaginosis in patients with koilocytosis. São Paulo Med. J. 126(6):333-336.

Carneiro D.O., Figueiredo H.C.P. \& Pereira Júnior D.J. 2007. Perfil de sensibilidade a antimicrobianos bactérias isoladas em diferentes sistemas de cultivo de tilápia-do-nilo (Oreochromis niloticus). Arq. Bras. Med. Vet. Zootec. 59:869-896.

CLSI 2006. Performace Standards for Antimicrobial Susceptibility Testing, $16^{\text {th }}$ Informational Supplement, Document M100-S16. Clinical and Laboratory Standards Institute, Wayne, PA, USA.

Coggan J.A., Melville P.A., Oliveira C.M., Faustino M., Moreno A.M. \& Benites N.R. 2008. Microbiological and histopathological aspects of canine pyometra. Braz. J. Microbiol. 39:477-483.

Drescher G., Matiello S.P., Peixoto R.M., Vargas A.C., Maciel M.N. \& Costa M.M. 2010. Caracterização bioquímica e perfil de sensibilidade aos antimicrobianos de agentes bacterianos isolados de mastite subclínica ovina na região oeste de Santa Catarina. Ciênc. Anim. Bras., Goiânia, 11(1):188-193.

Domingues P.F., Lucheis S.B., Serrão L.S., Fernandes S., Contente A.P.A., Martins, E.C.V. \& Langoni H. 2006. Etiologia e sensibilidade bacteriana da mastite subclínica em ovelhas da raça santa Inês. Ars Vet. 22(2):146-152.

Elliot L., McMahon K.J., Gier H.T. \& Marion G.B. 1968. Uterus of the cow after parturition: Bacterial content. Am. J. Vet. Res. 29(1):77-81.

Faubleé V.G., Tardy F., Bouveron C. \& Carret G. 2002. Antimicrobial susceptibility of Streptococcus species isolated from clinical mastitis in dairy cows. Int. J. Antimicrob. Agents 19:219-226.

Fornazari F., Ribeiro M.G., Santos F.C., Teixeira C.R., Rahal S.C., Teixeira R.H.F. \& Rassy F.B. 2007. Microbiota oral e sensibilidade microbiana in vitro em oito quatis criados no zoológico municipal Quinzinho de Barros, Sorocaba, SP. Ciênc. Vet. Trop. 10(2/3):108-112.

Freitas M.F.L., Pinheiro Júnior J.W., Stamford T.L.M,. Rabelo S.S.A., Silva D.R., Silveira Filho V.M., Santos F.G.B., Sena M.J. \& Mota R.A. 2005. Perfil de sensibilidade antimicrobiana in vitro de Staphylococcus coagulase positivos isolados de leite de vacas com mastite no agreste do estado de Pernambuco. Arqs Inst. Biológico, São Paulo, 72(2):171-177.

Fthenakis G.G. 1998. Susceptibility to antibiotics of staphylococcal isolates from cases of ovine or bovine mastitis in Greece. Small Rumin. Res. 28:9-13.

Gani M.O., Amin M.M., Alam M.G.S., Kayesh M.E.H., Karim M.R., Samad M.A. \& Islam M.R. 2008. Bacterial flora associated with repeat breeding and uterine infections in dairy cows. Bangl. J. Vet. Med. 6(1):79-86.

Gomes M.C. 2006. Microbiota vaginal de cabras nas fases do proestro, pós cópula e pós parto. Dissertação de Mestrado, Universidade Federal Rural do Rio de Janeiro, Seropédica, RJ. 39p.

Gregory L. \& Rizzo H. 2009. Atualidades no manejo das enfermidades reprodutivas. $4^{\circ}$ Congresso Internacional Feinco, São Paulo. Disponível em <http://www.sheepembryo.com.br/files/artigos/172.pdf> Acesso 21 fev. 2010.

Husted J.R. 2003. Bacterial and fungal organisms in the vagina of normal cows and cows with vaginitis. Dissertação de Mestrado. Veterinary Microbiology, Texas A. and M. University. 29p.

IBGE 2009. Sistema IBGE de Recuperação Automática (SIDRA). Disponível em <http://www.sidra.ibge.gov.br> Acesso 26 mai. 2009.

Jacob J.C.F., Jesus V.L.T., Barbosa H.P., Zimmerman M.F., Silva A.G. \& Melo C.M. 2002. Susceptibilidade antimicrobiana de $s w a b$ uterino e da fossa clitoriana de éguas com subfertilidade. Revta Univ. Rural, Sér. Ciênc. Vida 22(2):109-114.

Kreplin C.M. 1990. Infectious causes of reduced fertility in cattle. Alberta Agriculture, Food and Rural Devlopment 1:31.

Krumpermam P.H. 1983. Multiple antibiotic resistance indexing of Escherichia coli to identify high-risk sources of fecal contamination of food. Appl. Environ. Microbiol. 46(1):165-170.

Kuntze A. \& Aurich J. 1995. Der Endometritis-Pyometra-Komplex bei Tieren. Vet. Special, Gustav Fischer Verlag Jena.

Kunz T.L., Gambarini M.L., Oliveira Filho B.D. \& Galindo A.D.S. 2002. Morta- lidade embrionária em bovinos: inter-relações embrião-patógenos. Revta CFMV, Brasília, 8:28-36.

Lianjuan M., Yuemin L., Xun M. 1995. Microbial flora of the vagina of cows after parturition. Chin. J. Vet. Scienc. Technol. 25(5):7-26.

Livermore D.M. 1995. Beta-Lactamases in laboratory and clinical resistance, Clin. Microbiol. 8:557-584.

Lyon B.R. \& Skurray R. 1987. Antimicrobial resistance of Staphylococcus aureus: Genetic basis. Microbiol. 51:88-134.

Macedo N.R., Menezes C.P.L., Lage A.P., Ristow L.E., Reis A. \& Guedes R.M.C. 2007. Detecção de cepas patogênicas pela PCR multiplex e avaliação da sensibilidade a antimicrobianos de Escherichia coli isoladas de leitões diarréicos. Arq. Bras. Med. Vet. Zootec. 59:1117-1123.

Machado T.R.O., Correa M.G. \& Marin J.M. 2008. Antimicrobial susceptibility of coagulase-negative Staphylococci isolated from mastitic cattle in Brazil. Arq. Bras. Med. Vet. Zootec. 60(1):278-282.

Martins G., Figueira L., Penna B., Brandão F., Varges R., Vasconcelos C. \& Lilenbaum W. 2009. Prevalence and antimicrobial susceptibility of vaginal bactéria from ewes treated with progestin- impregnated intravaginal sponges. Small Rumin. Res. 81:182-184.

Moorthy A.R. \& Singh S.P. 1982. Studies on the bacterial flora of the female genital tract of sheep. Bull. Anim. Health Prod. Afr. 30(1):8-15.

Oliveira S.J. 1995. Guia Bacteriológico Prático. Ulbra, Canoas.

Panangala V.S., Fish N.A. \& Barnum D.A. 1978. Microflora of the cervicovaginal mucus of repeat breeder cows. Can. Vet. J. 19(4):83-89.

Pessoa C.A. 2009. Avaliação da microbiota bacteriana e fúngica presente na cloaca de jabutis (Geochelone carbonária) criados em domicílio e análise do potencial risco à saúde humana. Dissertação de Mestrado, USP, São Paulo. 97p.

Piddock L.J.V. 1996. Does the use of antimicrobial agents in veterinary medicine and animal husbandry select antibiotic resistant bacteria that infect man and compromise antimicrobial chemotherapy? J. Antimicrob. Chemother. 38:1-3.

Quinn P.J., Carter M.E., Markey B. \& Carter G.R. 1994. Clinical Veterinary Microbiology. $4^{\text {th }}$ ed. Wolfe, London. 648p.

Ramaswamy V., Andrew M. \& Roy P. 1991. Aerobic microbes of cervicovaginal mucus from repeat breeders bovines and their antibiogram. Sing. Vet. J. 14(15):60-65.

Riedner S., Albuquerque A.J.D., Badke M.R.T. \& Weiblen R.1987. Resistência de bactérias isoladas do leite de vacas frente a doze drogas antibacterianas. Revta Ciênc. Rural, Santa Maria, 11(3):251-260.

Rocha A.A., Gambarini M.L., Andrade M.A., Filho B.D.O. \& Gomes F.A. 2004. Microbiota cérvico-vaginal durante o final de gestação e puerpério em vacas girolando. Ciênc. Anim. Bras., Goiânia, 5(4):215-220.

Sena M.J. 2000. Perfil epidemiológico, resistência a antibióticos e aos conservantes nisina e sistema lactoperoxidase de Staphylococcus sp. isolados de queijos coalho comercializados em Recife-PE. Tese de Doutorado, Escola Veterinária, Universidade Federal de Minas Gerais, Belo Horizonte. 75 p.

Sharda R., Monghe M.N. \& Tanwani S.K. 1991. Antibiotic sensitivity pattern of bacteria isolated from repeat breeding animals. Indian Vet. J. 68:197-200.

Tabulsi I.R. 1999. Microbiologia. 3a ed. Ateneu, São Paulo.

Torres E.B., Nakao T., Hiramune T., Moriyoshi M., Kawata K. \& Nakada K. 1997. Stress and uterine bacterial flora in dairy cows following clinically normal and abnormal puerperium. J. Reprod. Develop. 43(2):157-163.

Torres C. \& Zarazaga M. 2002. Antibióticos como promotores del crescimiento en animales: vamos por el buen camino? Gac. Sanit. 16:109112.

Uchoa J.M.W.M.C., Silva N.G., Barreto M.B.P., Wischral A., Silva L.B.G., Batista A.M. \& Ferreira M.P.B. 2009. Flora bacteriana saprófita de fêmeas caprinas nulíparas criadas em regime de estabulação e pastoreio. IX Jornada de Ensino, Pesquisa e Extensão da UFRPE. Disponível <http://www. eventosufrpe.com.br/jepex2009/cd/resumos/R0472-1.pdf> Acesso 2 mar. 2010.

Verma H.K., Sharma D.K., Kaur H. \& Dhablama D.C. 1994. A bacteriological study of repeat breeders cows and their treatment. Indian Vet. J. 47(6):467-470.

Witte W. 1998. Medical consequences of antibiotics use in agriculture. Science 279:996-997.

Wunder J.A., Brinner W.W. \& Calkins G.P. 1976. Identification of the cultivable bacteria in dental plaque from the beagle dog. J. Dent. Res., Alexandria, 55(6):1097-1112. 